\title{
Development and Calibration of a Large-Scale Thermal Conductivity Probe
}

\author{
James L. Hanson,1 Stefan Neuhaeuser, 2 and Nazli Yesiller3
}

1 Associate Professor, Department of Civil Engineering, Lawrence Technological University, 21000 W. Ten Mile Rd., Southfield, MI 48075.

2 Assistant Project Engineer, Ruby and Associates, P.C., 30445 Northwestern Highway, Suite 310, Farmington Hills, MI 48334.

3 Associate Professor, Department of Civil and Environmental Engineering, Wayne State University, 5050 Anthony Wayne Dr., Detroit, MI 48202.

ABSTRACT: A large-scale probe has been developed for measuring the thermal conductivity of geomaterials. The large probe was designed to conduct tests on materials containing large particles, materials with high heterogeneity, and materials with high stiffness. The probe has dimensions of $680 \mathrm{~mm}$ length and $15.9 \mathrm{~mm}$ diameter and was constructed of stainless steel tubing. The probe operates on the principle of heating an infinite line source in an infinite medium. Initially, parametric evaluations were conducted to determine the operational and test conditions for the large probe, including power level, heating duration, and zone of heating influence. Then, tests were conducted on five homogeneous materials to calibrate the newly developed probe. Thermal conductivity measurements obtained using the large probe were compared with measurements obtained using a conventional small probe. A calibration curve was established for the large probe. In addition, the performance of the large probe was evaluated in two manufactured heterogeneous materials and a large particle material. The test program indicated that the large probe can be used effectively for determining thermal conductivity of geomaterials. This new probe may be suitable for large-scale laboratory testing and field investigations.

\section{Introduction}

Thermal conductivity of geomaterials needs to be known for analysis and evaluation of any engineering application involving heat transfer. Practical examples of thermal applications in geotechnical and geoenvironmental engineering include foundations for heated or cooled structures, buried high-power cables, underground space developments, various ground improvement applications, andwaste containment facilities. Thermal conductivity is needed for design that includes heat transfer applications as well as numerical analyses of heat transfer problems.

The needle probe method is used commonly to determine thermal conductivity, based on the theory of an infinite line source of heat in an infinite medium. The currently available needle probe equipment and test procedures have limitations. The volume influenced by heating is small and the probe is fragile, which makes insertion of the probe into firm materials difficult. Improvements are required to broaden the applicability of the method to heterogeneous materials, materials containing large particles, and firm 
materials. A largescale probe can be used to overcome the limitations of the small probe. The development, construction, and calibration of a largescale thermal conductivity probe are described in this paper. In addition, evaluation of the performance of the probe in various materials is presented.

\section{Background}

The analysis of thermal processes in geotechnical and geoenvironmental engineering and the corresponding design of engineering systems require knowledge of the thermal properties of geomaterials. Soils are used for heat dissipation or insulation for various geotechnical applications (Liu et al. 1989; Mihalakakou et al. 1994; Brandon et al. 1989; Lundy 1981; Moritz and Gabrielsson 2001; and Kuraoka et al. 1996). Thermal methods that induce temperature changes are used for ground improvement (Van Dijk and Vouwneester-van den Bos 2001; Hanson 1995; Meegoda et al. 2000; and Kosegi et al. 2000). In addition, thermal processes must be considered for frost heave (Andersland and Ladanyi 1994), landfill design (Doll 1997), and weather-dependent soil temperature variations (Farouki 1981).

There are three fundamental mechanisms responsible for heat propagation through materials: conduction, convection, and radiation. Conduction is by far the predominant mechanism for heat transfer in soils (Mitchell 1993). Thermal conductivity, $k$, quantifies the rate of heat flow through a material. The needle probe method can be used to determine thermal conductivity of a material and is based on the theory of temperature increase of an infinite linear constant heat source within an infinite medium. An analytical representation of this relationship is (Carslaw and Jaeger 1959):

$$
T=-\frac{q}{4 \pi k} E_{i}\left(-\frac{r^{2}}{4 \alpha t}\right)
$$

where

$$
\begin{aligned}
& T=\text { temperature increase }(\mathrm{K}) \\
& q=\text { heat input rate }(\mathrm{W} / \mathrm{m}) \\
& k=\text { thermal conductivity }(\mathrm{W} /[\mathrm{m} \cdot \mathrm{K}]) \\
& E i=\text { exponential integral } \\
& r=\text { radial distance from probe }(\mathrm{m}) \\
& \alpha=\text { thermal diffusivity }(\mathrm{m} 2 / \mathrm{s}) \\
& t=\text { time }(\mathrm{s}) \\
& \text { By expanding the exponential integral, Ei (Abramowitz and Stegun } 1964) \text { and }
\end{aligned}
$$
solving, it can be seen that the temperature increase, $T$, is linearly related to $\ln (t)$. The slope of this function, $s$, is:

$$
s=\frac{q}{4 \pi k}
$$

By rearranging Eq 2, the thermal conductivity, $k$, can then be determined as:

$$
k=\frac{q}{4 \pi s}
$$


The method described by Eqs 1 through 3 is applicable to experimental results, only if the theoretical assumptions are closely approximated. The thermal needle probe consists of a long cylindrical (needle-shaped) heater that approximates an infinite line heat source. A sufficiently large sample is necessary to simulate the infinite space and avoid boundary effects. A number of factors such as thermal instability, test material inhomogeneity and anisotropy, and uncertainty in determination of power input can affect the correct measurement of the thermal conductivity due to the deviation from the theoretical model (Nicolas et al. 1993).

A schematic graph of the typical temperature rise versus natural logarithm of time function for a thermal needle probe test is shown in Fig. 1 . The $T$ versus $\ln (t)$ graph contains three distinct portions. An initial transient portion is a response to the probe heating. The duration of this portion is dependent on the thermal properties of the probe and the contact resistance between the probe and the test material (Nicolas et al. 1993). The second portion of the curve is linear and represents a quasi-steady state condition for heat transfer from the probe. The slope of this linear portion of the curve represents the heat conduction through the medium under investigation and is used in Eq 3 to determine thermal conductivity. The boundary effects may cause a nonlinear trend (Fig. 1) at extended testing durations.

The needle probe method was standardized for soils and soft rock (ASTM D 5334, Method for Determination of Thermal Conductivity of Soil and Soft Rock by Thermal Needle Probe Method). Use of the thermal needle probe method for determining thermal conductivity of soils is well documented (Moench and Evans 1970; Mitchell and Kao 1978; Sepaskhah and Boersma 1979; Farouki 1981; Salomone et al. 1984; Salomone and Kovacs 1984; Brandon and Mitchell 1989; Nicolas et al. 1993; Hanson et al. 2000). Probe sizes can vary, but are commonly on the order of $100 \mathrm{mmlong}$ with a diameter of $1.8 \mathrm{~mm}$. Commercially available probes typically have similar dimensions.

A benefit of the thermal needle probe method is that testing duration and temperature rise are kept to a minimum, which limits heat-induced moisture migration that can affect thermal conductivity results for geomaterials (Farouki 1981). A limitation of a small probe is that it measures thermal conductivity only in the small zone of influence of the probe. If the sample has a high degree of spatial variability (containing individual materials with different thermal conductivity values), the probe measurements may give locally biased results with respect to the overall thermal conductivity. For earthen materials, this size limitation is present for large particle and/or spatially variable geomaterials, such as coarse aggregates, peats, and municipal solid waste. In addition, if a firm material is tested, the probe will have a tendency to bend upon insertion into the test material.

A potential method to overcome the limitations associated with the small thermal needle probe is the use of a large-scale probe. A number of large-scale thermal conductivity probes have been developed and reported for various applications. Reported dimensions of large probes range from 330 to $980 \mathrm{~mm}$ in length and from 9.5 to $15.9 \mathrm{~mm}$ in diameter. The quality of performance of the large probes has varied. Slusarchuk and Foulger (1973) developed a probe for measuring the thermal conductivity of permafrost. They obtained conductivity values within $4 \%$ of results obtained from guarded hot plate tests used as calibration. Jones (1988) developed a thermal probe for measuring thermal conductivity of coarse riverbed materials and obtained results within approximately $6 \%$ of reported 
values. Ewen and Thomas (1992) developed a probe to measure thermal conductivity and drying rate of soil in the field and obtained results within approximately $10 \%$ of reported values. Nicolas et al. (1993) developed a thermal probe for field testing and obtained results that were inconclusive.

The analysis of the large probe data presented has ranged from conventional thermal needle probe methods (Slusarchuk and Foulger 1973; Nicolas et al. 1993) to more sophisticated analyses with provisions for initial probe heating during the transient portion of the test (Jones 1988; Ewen and Thomas 1992). Analyzing the initial transient portion of the test shows promise for testing using a short heating duration while maintaining sufficient accuracy. However, this approach is complicated and requires either numerical modeling for comparison to the experimental data (Jones 1988) or specification of the probe/soil contact conductance and the ratio of the heat capacity of the soil to that of the probe for each test (Ewen and Thomas 1992). This approach may be justified for specialized applications; however, the extensive analysis required may not be practical for routine testing in a wide variety of geomaterials. In addition, the accuracy of thermal conductivity determined using these more complex methods is not superior to that obtained using conventional methods.

Details for construction and assembly of the large probes and thorough development of calibration procedures using parametric evaluations have not been widely reported. A systematic experimental analysis of the effects of power level, duration of testing, and influence radius has not been provided. In general, the materials in the reported test programs only included thermal conductivity values consistent with dry and saturated sands, with values between approximately 0.3 and $3 \mathrm{~W} /[\mathrm{m} \cdot \mathrm{K}]$. A greater variation of thermal conductivity exists for geomaterials (Hanson et al. 2000; Kaye and Laby 1973). The objective of this study was to design and construct a large-scale probe that allows for determination of thermal conductivity of a wide variety of materials using standard analysis methods, as outlined in Eqs 1-3. Construction of the probe is described in detail, followed by a description of calibration procedures for a wide range of thermal conductivity values. Performance of the probe in two manufactured heterogeneous materials and one large particle size material is then evaluated.

\section{Probe Construction}

The large probe was designed to provide a length:diameter aspect ratio sufficient to obtain idealized conditions of an infinite line source of heat $(680 \mathrm{~mm}$ length and $15.9 \mathrm{~mm}$ diameter). The aspect ratio for the probe was approximately 43 , whereas the aspect ratio for the standard small probe is approximately 55 (ASTM 2000). Other investigators have developed large probes with aspect ratios of approximately 35 (Slusarchuk and Foulger 1973), 47 (Ewen and Thomas 1992), and 71 (Nicolas et al. 1993).

The large probe consisted of a stainless steel outer tube, a stainless steel inner tube, a heating element, a thermocouple junction, thermal epoxy, and a stainless steel tip (Fig. 2). The outer tube was constructed of seamless stainless steel tubing $(680 \mathrm{~mm}$ long, $15.9 \mathrm{mmouter}$ diameter, and $0.9 \mathrm{~mm}$ wall thickness). The inner tube was constructed of welded stainless steel tubing ( $680 \mathrm{~mm}$ length, $6.25 \mathrm{~mm}$ outer diameter, and $1.24 \mathrm{~mm}$ wall thickness). The heating element consisted of heating tape made from nickel-chrome alloy resistance heating wire insulated with fiberglass cloth. The heating tape had a resistance of 128 _ and was $910 \mathrm{~mm}$ long, $12.5 \mathrm{~mm}$ wide, and $3 \mathrm{~mm}$ thick. The thermocouple wires were 
0.25-mm-diameter Type K (chromel/alumel) wires. A high conductivity thermal epoxy was used to fill the void spaces within the probe assembly. The tip had an apex of $60^{\circ}$ and was constructed of stainless steel.

Assembly of the probe components involved sequential placement of the thermocouple junction, thermal epoxy, heating tape, and probe tip. The thermocouple junction was placed at midlength inside the inner tube. Thermal epoxy was drawn up under vacuum using an apparatus as suggested in ASTM D 5334 to fill the remaining voids of the inner tube. The inner tube was then coated with epoxy. The heating tape was wrapped around the inner tube along a helical path. The wrapped assembly was coated with additional epoxy and inserted into the outer tube. The probe was assembled before the epoxy had cured to ensure distribution of the epoxy throughout the annular space. The probe was then allowed to cure. For testing, the probe was connected to a power supply and data acquisition system.

\section{Test Materials}

Tests were conducted using both the small and the large probes in materials spanning more than two orders of magnitude of thermal conductivity. The range selected is representative of the range of thermal conductivity for geomaterials.

Thermal conductivity tests were conducted on five homogeneous materials for determining testing conditions and operational parameters for the large probe, as well as calibration of the probe. These materials were: extruded polystyrene (XPS), dry Ottawa sand, glycerol, water, and saturated Ottawa sand. Water was solidified using $0.5 \%$ agar by weight to prevent moisture migration in the tests (water tests and saturated Ottawa sand tests).

Thermal conductivity tests were conducted on three materials to assess the performance of the large probe in heterogeneous and large particle materials. The heterogeneous materials consisted of mixtures of the relatively high $k$ material (Ottawa sand) and relatively lowk material (XPS). The XPSwas cut into pieces of approximately 100 $\mathrm{mm} \times 100 \mathrm{~mm} \times 50 \mathrm{~mm}$ and placed randomly throughout the sand. The first mixture consisted of XPS pieces mixed with dry Ottawa sand. The second mixture consisted of XPS pieces mixed with saturated Ottawa sand (saturated with water solidified using agar $0.5 \%$ by weight). The XPS contentwas $25 \%$ by volume for dry sand/XPS mixture and $50 \%$ by volume for the saturated sand/XPS mixture. The large particle material consisted of wood blocks (pressure treated pine) with approximate dimensions of $90 \times 90 \times 130 \mathrm{~mm}$. The blocks were placed with a porosity of approximately $50 \%$. A summary of test materials and sample dimensions is presented in Table 1.

\section{Preliminary Tests}

Prior to development of operational parameters and test conditions, as well as calibration of the large probe, two sets of tests were conducted. First, the accuracy of the small probe was validated since this probe was used to provide baseline values for calibration of the large probe. Tests were conducted on materials with reported thermal conductivity. The results of the verification tests are presented in Table 2. The thermal conductivities measured with the small probe for glycerol and water were within $5 \%$ of the reported values, whereas a greater deviation was obtained for dry Ottawa sand (within 
$12 \%$ of reported value). Although deviation from a reported value was greater for Ottawa sand, the measurements were highly repeatable (as reported below). Granular materials are inherently susceptible to variation of structure, even when density is controlled. In addition, the thermal conductivity of Ottawa sand is not as widely reported as the other calibration materials. The overall agreement between the small probe tests and the published values indicated that the small probe provided representative values and that it could be used as a reference for the calibration of the large probe.

Second, the uniformity of the calibration test materials was verified using two sets of tests: thermal conductivity was measured at 20 different locations, and 30 repeated measurements were taken at a single location in dry Ottawa sand. The results of these tests indicated that the variability of the measured $k$ at different locations (average absolute deviation from the mean, $\alpha$ was $3.4 \%$ )was somewhat greater than the variability associated with repeated tests at a single location ( $\alpha$ was $0.9 \%$ ). These results indicate that Ottawa sand is acceptable as a calibration medium for thermal conductivity testing as the variability is within the 5-10\%overall accuracy of the thermal needle probe method (Nicolas et al. 1993; ASTM 2000). The uniformity tests were not conducted for glycerol, water, or extruded polystyrene, as these materials are inherently homogeneous and their structures are not a function of sample preparation as is the case with Ottawa sand.

\section{Operational and Test Conditions for Large Probe}

Parametric evaluations were conducted to determine the operational and test conditions for the large probe. The effects of voltage applied to the probe and the duration of heating were investigated to establish the test conditions required to obtain representative thermal conductivity measurements. The evaluation of the zone of heating influence is used to determine the minimum size of samples required for the large probe tests. These tests were conducted on the homogeneous test materials. All test specimens were surrounded with thermal insulation to minimize the effects of external temperature variations.

\section{Power Level}

Tests were conducted to evaluate the temperature rise of the probe at various power levels. Three heat input rates $(q)$ were applied to investigate the effect of power level on probe response: $1.94 \mathrm{~W} / \mathrm{m}, 7.12 \mathrm{~W} / \mathrm{m}$, and $10.55 \mathrm{~W} / \mathrm{m}$, resulting from $12 \mathrm{~V}$ (voltage regulator/ deep cycle battery), $25 \mathrm{~V}$ (voltage converter), and $30 \mathrm{~V}$ (voltage converter) power supplies, respectively. The temperature rise of the probe at these three heat input rates is presented in Fig. 3 for dry Ottawa sand. It was observed that the temperature rise of the probe was a function of the heat input rate. At each power level the initial transient period was followed by a linear portion of $T$ versus $\ln (t)$. The arrival time of the linear portion of the $T$ versus $\ln (t)$ relationship is denoted $t a$. The thermal conductivity values at each power level were determined to be similar $( \pm 3 \%)$ because the slope of the linear portion of $T$ versus $\ln (t)$ was proportional to the heat input rate (i.e., as the heat input rate increased, the slope became steeper). Similar trends were observed for all the test materials. The power level for testing was selected to obtain a well-defined linear $T$ versus $\ln (t)$ trend that allowed for determining representative thermal conductivity values. 
Overheating during a test can affect the properties of test materials that contain water by causing moisture migration. Therefore, the power level was also selected for each material to provide a desirably small, yet measurable increase in temperature (0.5-3 K) upon heating. These two criteria (well-defined slope and small temperature rise) resulted in the selection of a 12-V nominal power supply for the homogeneous test materials, except for saturated sand. A higher power level was required for the saturated sand (18-V nominal power supply) to generate sufficient temperature rise in this high thermal conductivity material to establish fully the linear portion of $T$ versus $\ln (t)$.

The power supply selectedwas a voltage regulator with a nominal voltage of $12 \mathrm{~V}$. A high capacity $12-\mathrm{V}$ battery was connected in parallel to the regulator to dampen small fluctuations in voltage supply by the regulator and to provide power in case of power outage. This power supply system provided a constant voltage of $13 \mathrm{~V}$ and a heat input rate, $q$, of $1.94 \mathrm{~W} / \mathrm{m}$. The power supply was continuously monitored using a digital multimeter to assure consistency during each test. An advantage of this power level is that various nominal $12-\mathrm{V}$ power supplies are commonly available (e.g., car electrical system) to allowfor portability and field use. For the tests in saturated sand, the power supply was a nominal 18-V voltage converter, which provided a constant voltage of $19.5 \mathrm{~V}$ and a $q$ of 4.37 $\mathrm{W} / \mathrm{m}$.

In addition, analysis of the results of the power level tests demonstrated that $t a$ was essentially independent of power level for a particular material (Fig. 3). This is because transient heat transfer is a diffusive process (Mitchell 1993). For testing purposes, this indicates that a higher heat input rate will require the same duration test as a lower heat input rate. The duration of the tests must be selected to obtain a well-defined linear $T$ versus $\ln (t)$ relationship.

\section{Duration of Test}

Tests conducted using variable heating duration were evaluated to determine the minimum required heating duration. Heating duration should be minimized to prevent potential moisture migration. The linear $T$ versus $\ln (t)$ relationship was not well established at short heating durations. A constant value for $k$ (termed kultimate) is approached with increasing heating duration, as a well-established linear portion of $T$ versus $\ln (t)$ is obtained in the measurements. The conductivity measured for any heating duration is termed kmeasured. The relationship between heating duration and corresponding ratio of kmeasured to kultimate is presented for all homogeneous test materials in Fig. 4. The graph indicates that a longer heating duration is required to establish kultimate for the lowest $k$ material (XPS). A heating duration of approximately $1200 \mathrm{~s}$ is sufficient to establish a well-defined linear portion of the $T$ versus $\ln (t)$ relationship for the large probe in glycerol, dry sand, water, and saturated sand, whereas a heating duration of approximately $3000 \mathrm{~s}$ is required for XPS (Fig. 4). Large probe tests on all materials used a heating duration of $3600 \mathrm{~s}$ to provide consistency throughout the test program and to satisfy minimum required duration for all test materials (Fig. 4). The 3600$s$ heating duration did not cause moisture migration in the test materials. Testing natural materials in the field may require verification of testing duration to prevent moisture migration. 
The relative increase in temperature rise at distance from the probe, with respect to temperature rise of the probe, is required to determine the radial extent of heating within a sample. Thermocouple sensors were placed at incremental radial distances from the probe in XPS, dry Ottawa sand, and saturated Ottawa sand. These three materials were selected to represent the entire range of thermal behavior (low, high, and intermediate $k$ ), while avoiding potential convective heat transfer associated with measurements in the less viscous test materials, water and glycerol. The zone of heating influence is evaluated by measuring $T$ at various distances from the probe within large samples of the test materials. The sample sizes used were: $850 \mathrm{mmlength} \times 540 \mathrm{mmdiameter}$ for dry and saturated Ottawa sand, and $1000 \times 300 \times 300 \mathrm{~mm}$ for XPS.

Typical results of temperature rise as a function of distance from the probe for dry Ottawa sand using a heating duration of $3600 \mathrm{~s}$ are presented in Fig. 5. Figure 5 shows that temperature rise diminishes with distance from the probe. In addition, arrival time of the initial temperature increase is delayed with increasing distance from the probe. The maximum temperature rises measured were approximately $0.42 \mathrm{~K}$ at $25 \mathrm{~mm}$ and $0.20 \mathrm{~K}$ at $50 \mathrm{~mm}$. Temperature rise, $T$, measured at radial distances greater than $50 \mathrm{~mm}$ from the probe over $3600 \mathrm{~s}$ of heating duration was negligible in the dry Ottawa sand. Similar trends were obtained for the other test materials; however, the extent of temperature rise varied with material (Fig. 6 and Table 3).

The heating zone in various materials was compared using the ratio of temperature rise at a radial distance from the probe to the corresponding temperature rise of the probe at any given time $(\delta)$, [Fig. 6 and Table 3]. Comparisons were made for data obtained for a 3600 -s heating duration at various distances from the probe to determine the minimum required sample sizes.

First, $\delta$ is compared at a distance $50 \mathrm{~mm}$ from the probe in the three materials for a heating duration of $3600 \mathrm{~s}$ (Fig. 6).Adistance of $50 \mathrm{~mm}$ was selected for this comparison as measurable temperature rise occurred in all three test materials at this distance. A high relative temperature rise was measured in saturated sand and dry sand

$(\delta=0.08)$, and a lowrelative temperature risewas measured in XPS $(\delta=0.02)$ at this distance. Next, $\delta$ is compared at various distances from the probe in the three materials for a heating duration of 3600 s (Fig. 6). The relative temperature rise extended further outward from the probe in saturated sand, compared to XPS and dry sand. The smaller $\delta$ value for XPS indicates that the majority of heat energy was contained in the near vicinity of the probe, whereas in the sand samples (higher $\delta$ ), the heat energy had spread to a greater volume of the test sample. Similar trends were reported in previous studies indicating that zone of heating influence is a function of thermal diffusivity (Slusarchuk and Foulger 1973; Brandon and Mitchell 1989; Nicolas et al. 1993). The volume of material heated (and therefore tested) for a given heat input (duration and intensity) is greater for high thermal diffusivity materials, such as wet soils, than for low thermal diffusivity materials, such as light dry materials (Lundy 1981).

Boundary effects may cause a divergence from a linear $T$ versus $\ln (t)$ relationship, which in turn may affect determination of $k$. Various investigators have identified an analytical relationship indicating that $\delta$ should be lower than 0.02 at the boundary of the test samples (Slusarchuk and Foulger 1973; Brandon and Mitchell 1989; Nicolas et al. 1993). However, experimental verification for this approach has not been provided. 
Tests were conducted to assess the applicability of the analytical $\delta(0.02)$ for the large probe. No divergence in the linear $T$ versus $\ln (t)$ relationship was observed when $\delta$ exceeded 0.02 in various materials. Similar $k$ values were obtained for $\delta=0.05,0.08$, and 0.15 in saturated sand, XPS, and dry sand, respectively. Based on these findings, a practical limit of $\delta=0.05$ was established to provide empirical recommendations for sample sizes. The trends presented in Fig. 6 were used to determine sample sizes corresponding to $\delta=$ 0.05 . The minimum sample sizes for saturated sand, dry sand, and XPS are determined to be $260 \mathrm{~mm}, 150 \mathrm{~mm}$, and $50 \mathrm{~mm}$ diameter, respectively. It is expected that the intermediate materials in terms of thermal behavior (water and glycerol) would require intermediate sample sizes. Radial dimensions of samples used for the test program (Table 1) exceeded the minimum required dimensions. The temperature rises at the boundary of the samples were measured during the tests to confirm that $\delta$ remained below 0.05 at the sample boundaries.

\section{Calibration Tests}

Subsequent to determination of the testing conditions and operational parameters for the large probe, tests were conducted to calibrate the probe using measurements obtained with the small probe. The probe was calibrated using tests conducted in five homogeneous materials. Multiple tests were conducted with both probes in each test material to assess repeatability of test results and provide correlation between the two sets of tests. Each series of tests in a material consisted of 30 measurements using the small probe and 30 measurements using the large probe.

Results of the test program comparing the behavior of small and large probes are presented in Fig. 7 and Table 4. The coefficient of variation within each series of tests is $5 \%$ or less for all materials tested with both probes, except for saturated sand. Because of the relatively small temperature rise generated during tests in saturated sand, minor external temperature fluctuations have a greater impact on the test results. These effects may be the cause for the increased variability of the test results in saturated sand. The smallest level of variability was observed for dry sand for both probes. This small variation may be attributed to the larger sample size that was used for the dry sand than for the other materials. The greater sample volume tends to decrease any effects of minor laboratory temperature fluctuations. For all materials tested, the variability of the test results was slightly greater for the large probe than for the small probe. The added level of variability may be due to longer test durations and the potential heterogeneity associated with the larger volume of heated material. Nevertheless, the variability in the tests conducted using both probes was generally within the accepted level of precision for thermal probe method, approximately 5-10 \% (ASTM 2000).

The large probe measurements (klarge) were correlated to baseline values obtained from the small probe (ksmall) using a calibration factor. The calibration factor, $F$, is defined as ksmall/klarge. Good agreement was observed between the results from the large probe and the small probe for tests conducted on water $(F=0.99)$. For the test material with thermal conductivity greater thanwater (saturated sand), a lower apparent thermal conductivity value is measured with the large probe than with the small probe (Table 4). For the test materials with thermal conductivity less thanwater (glycerol, dry sand, and XPS) a higher apparent thermal conductivity value is measured with the large probe than with the small probe, corresponding to calibration factors less than 1.0 (Table 4). 
A calibration curve was developed using a plot of thermal conductivity measurements (obtained from the large probe) versus calibration factor (Fig. 8). A logarithmic scale was used for thermal conductivity, since a wide range of values was included in the plot. The resulting log-linear relationship is described by Eq 4.

$F=0.1641 \cdot \ln \left(k_{\text {large }}\right)+1.0439(4)$

The calibration factor, $F$, can be used to calculate the corrected thermal conductivity, kcorrected, as indicated in Eq 5.

$k_{\text {corrected }}=F \cdot k_{\text {large }}(5)$

The value of $R 2=0.9677$ obtained from the calibration curve indicates that the individual calibration factors over the tested range of $k$ are well correlated to the calibration function of Eq 4.

\section{Performance Tests}

A series of tests was conducted to assess the effectiveness of the large probe in measuring the thermal conductivity of heterogeneous and large particle materials. Tests were conducted in samples containing heterogeneous mixtures of XPS and sand (both dry and wet), and also in a sample of large wood blocks.

For heterogeneous materials tests, measurements were taken using the small probe at various locations throughout the mixtures to assess the variability of measured $k$. Twelve measurements were conducted at three different locations (36 total tests): within the sand matrix, within a single piece of XPS, and at an interface of XPS and sand. For the dry Ottawa sand/XPS mixture, an additional series of twelve tests was conducted with half of the length of thesmall probe surrounded by sand and half of the probe surrounded by XPS. Then, twelve tests were conducted using the large-scale probe at a central location within the samples to assess whether a representative $k$ would be measured.

Results of these tests are presented in Figs. 9 and 10. The large probe values were corrected using the calibration factor, $F$ (Fig. 8). For dry Ottawa sand/XPS mixture, an average corrected $k$ of $0.192 \mathrm{~W} /[\mathrm{m} \cdot \mathrm{K}]$ was obtained using the large probe, whereas the small probe measurements ranged from 0.013 to $0.25 \mathrm{~W} /[\mathrm{m} \cdot \mathrm{K}]$, depending on placement location (Fig. 9). For the saturated Ottawasand/XPS mixture, an average corrected $k$ of 1.08 $\mathrm{W} /[\mathrm{m} \cdot \mathrm{K}]$ was obtained using the large probe, whereas the small probe measurements ranged from 0.019 to $2.51 \mathrm{~W} /[\mathrm{m} \cdot \mathrm{K}]$, depending on placement location (Fig. 10).

An analysis was conducted to estimate $k$ for the two heterogeneous mixtures. An overall $k$ was calculated for the heterogeneous materials using a weighted average of the $k$ of the individual components of the mixtures (sand and XPS). Volumetric fractions of each component were used as the weighting factors. This analysis resulted in $k=0.165 \mathrm{~W} /[\mathrm{m}$. $\mathrm{K}]$ for the $75 \%$ dry Ottawa sand $/ 25 \%$ XPS mixture and $k=1.26 \mathrm{~W} /[\mathrm{m} \cdot \mathrm{K}]$ for the $50 \%$ saturated Ottawa sand $/ 50 \%$ XPS mixture. These values demonstrate reasonable agreement with $k$ measured using the large probe. The measured $k$ values varied by 14.3 and $17.1 \%$ from the theoretical approximations for dry Ottawa sand/XPS and saturated Ottawa sand/XPS, respectively. It must be noted that this idealized analysis is simplistic and does not account for the actual spatial placement of the components of the mixtures in 
the vicinity of the probe. It is believed that the variation between the measured large probe $k$ and the weighted average $k$ is acceptable, and that the large probe measurements captured the thermal behavior of the heterogeneous materials.

Similar to the heterogeneous materials tests, measurements were conducted in the large particle (wood blocks) sample with both the small and large probes (Fig. 11). Twelve measurements were conducted at three different locations with the small probe (36 total tests): within the voids, directly between wood blocks, and at an interface of wood and air. Then, twelve tests were conducted using the large probe at a central location within the sample to assess whether a representative $k$ would be measured. An average corrected $k$ of $0.088 \mathrm{~W} /[\mathrm{m} \cdot \mathrm{K}]$ was measured using the large probe. Small probe thermal conductivity measurements ranged from 0.020 to $0.18 \mathrm{~W} /[\mathrm{m} \cdot \mathrm{K}]$, depending on placement location. A weighted average analysiswas conducted to estimate $k$ for thewood blocks, using the individual $k$ of the components (air and wood) of the sample. This analysis resulted in $k=$ $0.100 \mathrm{~W} /[\mathrm{m} \cdot \mathrm{K}]$ for the wood blocks. The measured $k$ value varied by $11.8 \%$ from the theoretical approximation. The test results indicated that the large probe accounted for the global structure of the large particle test material.

\section{Summary and Conclusions}

A large-scale thermal needle probe was constructed to conduct thermal conductivity tests on geomaterials. The large probe was developed to overcome the limitations of the small needle probe, namely, the small volume of material tested and the flexibility of the small probe. The large probe was designed for testing materials containing large particles, materials with high heterogeneity, and materials with high stiffness using conventional thermal needle probe theory.

Parametric evaluations were conducted to determine the operational and test conditions for the large probe. The effects of voltage applied to the probe and the duration of heating were investigated to establish the test conditions required to obtain representative thermal conductivity measurements. Analysis of the zone of heating is used to determine the minimum sample sizes required for the large probe tests.

Calibration tests were conducted using a conventional small probe in five homogeneous materials that spanned more than two orders of magnitude of thermal conductivity $(0.012-2.88 \mathrm{~W} /[\mathrm{m} \cdot \mathrm{K}])$. The range selected was representative of the range of thermal conductivity for geomaterials. The measurements obtained using the small probe were taken as baseline values, and an empirical correction relationship was developed to calibrate the large probe using these values. The probe construction, development, and calibration resulted in:

\section{Probe}

Dimensions—680 mm long $\times 15.9 \mathrm{~mm}$ diameter

Materials and Construction-Stainless steel tubing, fiberglass wrapped heating tape, thermal epoxy, Type $\mathrm{K}$ thermocouple, pointed tip, and rigid construction for placement into stiff materials.

\section{Operational and Test Conditions}


Power Level-12 V ( $k \leq 0.6 \mathrm{~W} /[\mathrm{m} \cdot \mathrm{K}])$, higher level (18 V) may

be needed for high conductivity material $(k=2.9 \mathrm{~W} /[\mathrm{m} \cdot \mathrm{K}])$

Heating Duration-3600 s

Sample Size-Material dependent, 50mmfor low $k$, light and dry materials (e.g., XPS) to $260 \mathrm{~mm}$ diameter for high $k$, heavy and wet materials (e.g., saturated sand).

\section{Calibration}

Thermal conductivity from large probe, kcorrected $=F \cdot$ klarge $F=0.1641 \cdot \ln ($ klarge $)+1.0439(\mathrm{R} 2=0.9677)$

In addition, performance tests were conducted to evaluate the effectiveness of the newly developed large probe for testing heterogeneous and large particle materials. It was observed that representative measurements were obtained on these materials using the large probe. The probe may be suitable for testing large particle, heterogeneous, and stiff materials both in the laboratory and in the field.

Acknowledgment

Funding for this project was provided by the National Science Foundation (Grant No. CMS9813248). Mr. Ray Ziegler assisted with fabrication of the large-scale thermal needle probe at Lawrence Technological University.

\section{References}

ASTM Standard, 2000, C 778-92: Specification for Standard Sand, Annual Book of ASTM Standards,Vol. 4.01,ASTMInternational, West Conshohocken, PA.

ASTM Standard, 2000, D 5334-92: Test Method for Determination of Thermal Conductivity of Soil and Soft Rock by Thermal Needle Probe Procedure, ASTM Standards on DISC, Volume 04.08, ASTM International, West Conshohocken, PA.

Abramowitz, M. and Stegun, I., Eds., 1964, Handbook of Mathematical Functions with Formulas, Graphs, and Mathematical Tables, 9th Printing, United States of America Department of Commerce, National Bureau of Standards, Applied Mathematics Series 55, Washington, D.C.

Andersland, O. B. and Ladanyi, B., 1994, An Introduction to Frozen Ground Engineering, Chapman and Hall, New York, NY.

Brandon, T. L. and Mitchell, J. K., 1989, “Factors Influencing Thermal Resistivity of Sands,” Journal of Geotechnical Engineering, ASCE, Vol. 115, No. 12, pp. 1683-1698.

Brandon, T. L., Mitchell, J. K., and Cameron, J. T., 1989, “Thermal Instability in Buried Cable Backfills," Journal of Geotechnical Engineering, ASCE, Vol. 115, No. 1, pp. 38-55. 
Carslaw, H. S. and Jaeger, J. C., 1959, Conduction of Heat in Solids, 2nd ed., Oxford University Press, London.

Doll, P., 1997, “Desiccation of Mineral Liners Below Landfills with Heat Generation,” Journal of Geotechnical and Geoenvironmental Engineering, ASCE, Vol. 123, No. 11, pp. 1001-1009.

Ewen, J. and Thomas, H. R., 1992, “The Thermal Probe- Measurement of the Thermal Conductivity and Drying Rate of Soil in the Field," Geotechnical Testing Journal, Vol. 15, No. 3, pp. 256-263.

Farouki, 0. T., 1981, Thermal Properties of Soils, Cold Regions Research and Engineering Laboratory Report 82-8, U.S. Army Corps of Engineers, Hanover, NH.

Hanson, J. L., 1995, “Field Demonstration of Thermal Precompression of Peat," Proceedings, 43rd Annual Geotechnical Engineering Conference, University of Minnesota, Minneapolis, MN, pp. 1-16.

Hanson, J. L., Edil, T. B., and Yesiller, N., 2000, “Thermal Properties of High Water Content Materials," Geotechnics of High Water Content Materials, ASTM STP 1374, T. B. Edil and P. J. Fox, Eds., ASTM International, West Conshohocken, PA, pp. 137-151.

Jones, B.W., 1988, “Thermal Conductivity Probe: Development of Method and Application to a Coarse Granular Medium," Journal of Physics E, Scientific Instruments, IOP Publishing, Vol. 21, pp. 832-839.

Kaye, G.W. C. and Laby, T. H., 1978, Table of Physical and Chemical Constants and Some Mathematical Functions, Longman, New York, NY.

Kosegi, J. M., Minsker, B. S., and Dougherty, D. E., 2000, “Feasibility Study of Thermal In Situ Bioremediation,” Journal of Environmental Engineering, ASCE, Vol. 126, No. 7, pp. 601-610.

Kuraoka, S., Rajani, B., and Zhan, C., 1996, "Pipe-Soil Interaction Analysis of Field Tests of Buried PVC Pipes,” Journal of Infrastructure Systems, ASCE, Vol. 2, No. 4, pp. 162-170.

Liu, S., Soong, K., and Yang, J., 1989, "Final Disposal of Spent Nuclear Fuel in Taiwan: A State-of-the-Art Technical Overview," Waste Management, Elsevier Science, Vol. 9, No. 3, pp. 171-188.

Lundy, K., 1981, Regional Analysis of Ground and Above-Ground Climate, Oak Ridge National Laboratory, Oak Ridge, TN.

Meegoda, J. N., Partymiller, K., Richards, M. K., Kamolpornwijit, W., Librizzi, W., Tate, T., Noval, B. A., Mueller, R. T., and Santora, S., 2000, "Remediation of Chromium-Contaminated Soils-Pilot-Scale Investigation," Practice Periodical of Hazardous, Toxic, and Radioactive Waste Management, ASCE, Vol. 4, No. 1, pp. 7-14. 
Mihalakakou, G., Santamouris, M., and Asimakopoulos, D., 1994, "Use of the Ground for Heat Dissipation," Energy, Elsevier Science, Vol. 19, No. 1, pp. 17-25.

Mitchell, J. K., 1993, Fundamentals of Soil Behavior, John Wiley and Sons, New York.

Mitchell, J. K. and Kao, T. C., 1978, “Measurement of Soil Thermal Resistivity,” Journal of the Geotechnical Engineering Division, ASCE, Vol. 104, No. GT10, pp. 1307-1320.

Moench, A. F. and Evans, D. D., 1970, "Thermal Conductivity and Diffusivity of Soil Using a Cylindrical Heat Source," Soil Science Society of America Proceedings, Soil Science Society of America, Vol. 34, pp. 377-381.

Moritz, L. and Gabrielsson, A., 2001, “Temperature Effect on the Properties of Clay," Soft Ground Technology, GSP No. 112, ASCE, J. L. Hanson and R. J. Termaat, Eds., pp. 304-314.

Nicolas, J., Andr'e, P., Rivez, J. F., and Debbaut, V., 1993, “Thermal Conductivity Measurements in Soil Using an Instrument Based on the Cylindrical Probe Method," Review of Scientific Instruments, Vol. 64, No. 3, pp. 774-780.

Salomone, L. A. and Kovacs,W. D., 1984, “Thermal Resistivity of Soils,” Journal of Geotechnical Engineering, ASCE, Vol. 110, No. 3, pp. 375-389.

Salomone, L. A., Kovacs, W. D., and Kusuda, T., 1984, “Thermal Performance of Fine-Grained Soils," Journal of Geotechnical Engineering, ASCE, Vol. 110, No. 3, pp. 359-374.

Sepaskhah, A. R. and Boersma, L., 1979, “Thermal Conductivity of Soils as a Function of Temperature and Water Content," Soil Science of America Journal, Soil Science Society of America, Vol. 43, No. 3, pp. 439-444.

Slusarchuk,W. A. and Foulger, P. H., 1973, Development and Calibration of a Thermal Conductivity Probe Apparatus for Use in the Field and Laboratory, Technical Paper No. 388 of the Division of Building Research, National Research Council of Canada,Ottawa.

Van Dijk, P. and Vouwneester-van den Bos, J., 2001, "Large Scale Application of Artificial Ground Freezing," Soft Ground Technology, GSP No. 112, ASCE, J. L. Hanson and R. J. Termaat, Eds., pp. 315-330.

Vargaftik, N. B., 1975, Tables on the Thermophysical Properties of Liquids and Gases, 2nd ed., Hemisphere Publishing Corporation,Washington, D.C. 


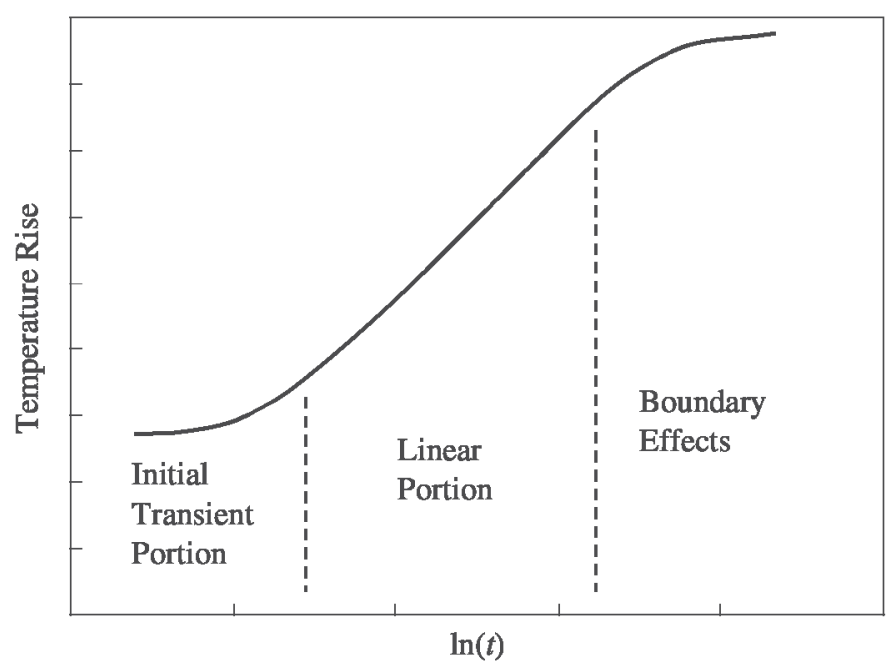

FIG. 1-Typical thermal conductivity test results.

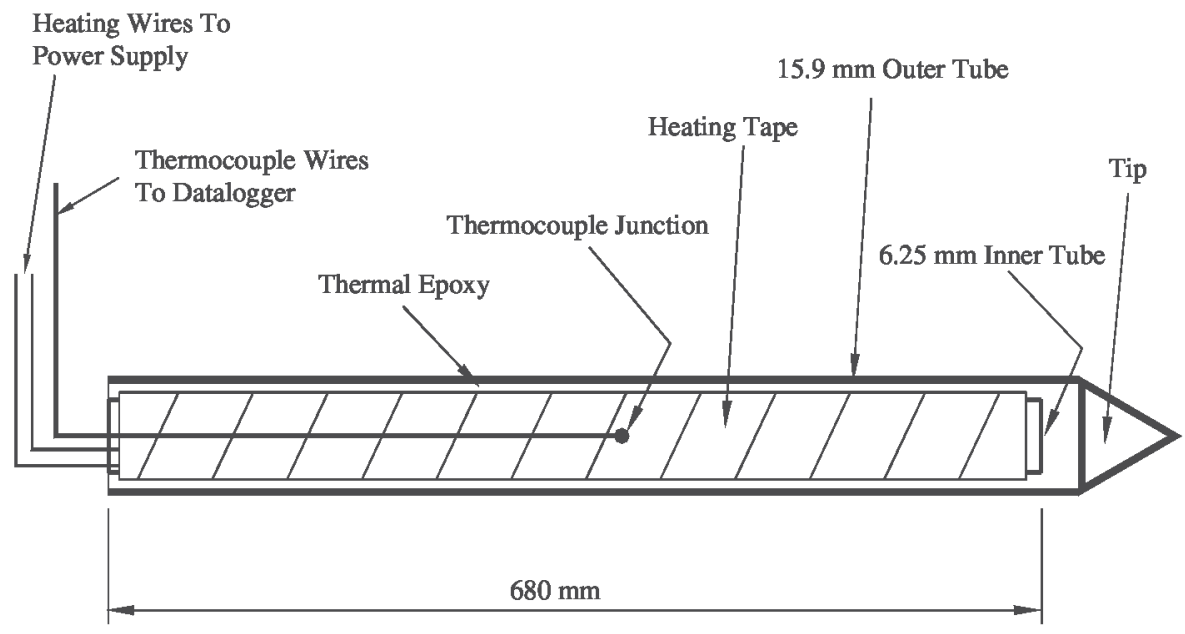

FIG. 2 Schematic of large probe.

TABLE 1-Materials tested.

\begin{tabular}{lcc}
\hline \multicolumn{1}{c}{ Material } & $\begin{array}{c}\text { Unit Weight, } \\
\mathrm{kN} / \mathrm{m}^{3}\end{array}$ & Sample Dimensions, mm \\
\hline Extruded Polystyrene (XPS) & 0.244 & $1000 \times 150 \times 150$ \\
Dry Ottawa Sand $^{1}$ & 16.20 & $850 \times 540$ (diameter) \\
96\% Pure Glycerol & 12.40 & $1050 \times 300$ (diameter) \\
Water $^{2}$ & 9.81 & $1050 \times 300$ (diameter) \\
Saturated Ottawa Sand $^{1,2}$ & 20.00 & $1050 \times 300$ (diameter) \\
$\begin{array}{l}\text { Dry Ottawa Sand } \\
\quad \text { Mixture }\end{array}$ & 12.20 & $1050 \times 300$ (diameter) \\
$\begin{array}{l}\text { Saturated Ottawa Sand } \\
\quad \text { Mixture }\end{array}$ & 10.10 & $1050 \times 300$ (diameter) \\
\begin{tabular}{l} 
Wood Blocks \\
\hline
\end{tabular} & 3.25 & $850 \times 540$ (diameter) \\
\hline
\end{tabular}

${ }^{1}$ ASTM C 778-Specification for Standard Sand, Grade 20-30 (ASTM 2000).

${ }^{2}$ Solidified using agar, $0.5 \%$ by weight. 
TABLE 2-Small probe verification test results.

\begin{tabular}{cccl}
\hline & $\begin{array}{c}\text { Average } \\
k_{\text {measured }}, \\
\mathrm{W} /[\mathrm{m} \cdot \mathrm{K}]\end{array}$ & $\begin{array}{c}k_{\text {reported, }} \mathrm{W} /[\mathrm{m} \cdot \mathrm{K}] \\
\text { Material }\end{array}$ & \multicolumn{1}{c}{ Reference } \\
\hline $\begin{array}{c}\text { Glycerol } \\
\text { Water solidified with } \\
0.5 \% \text { agar }\end{array}$ & 0.293 & 0.286 & Kaye and Laby (1978) \\
Dry Ottawa Sand & 0.581 & 0.602 & Vargaftik (1975) \\
& 0.262 & $0.304^{2}$ & $\begin{array}{c}\text { Slusarchuk and Foulger } \\
\text { (1973) }\end{array}$ \\
\hline
\end{tabular}

${ }^{1}$ Prepared to $16.6 \mathrm{kN} / \mathrm{m}^{3}$ unit weight.

${ }^{2}$ Using guarded hot plate method.

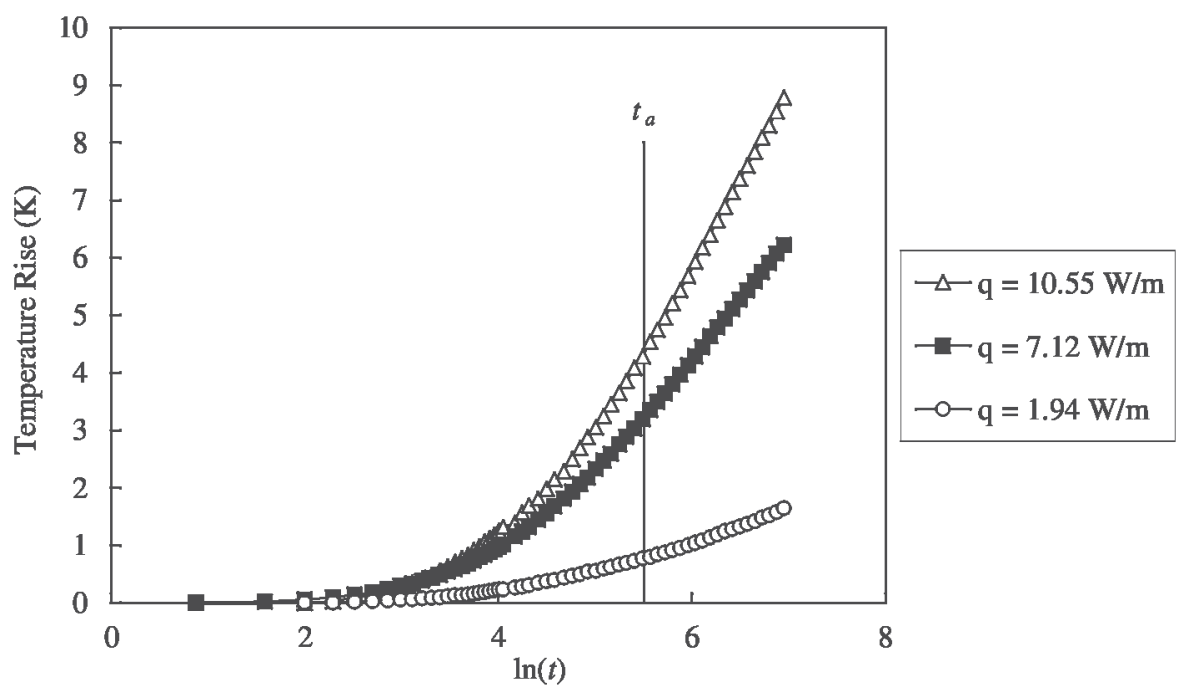

FIG. 3-Effect of power level on large probe measurements (dry sand). 


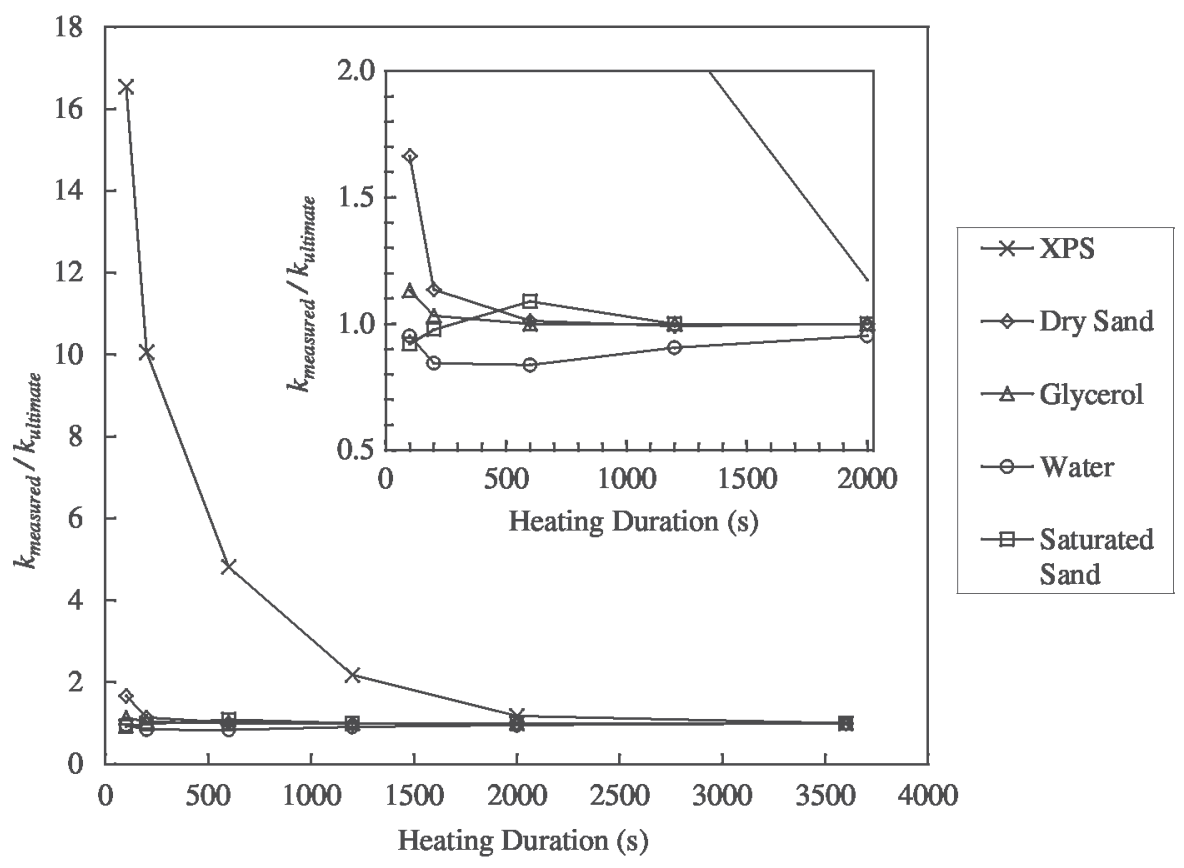

FIG. 4 Effect of heating duration (large probe in various test materials).

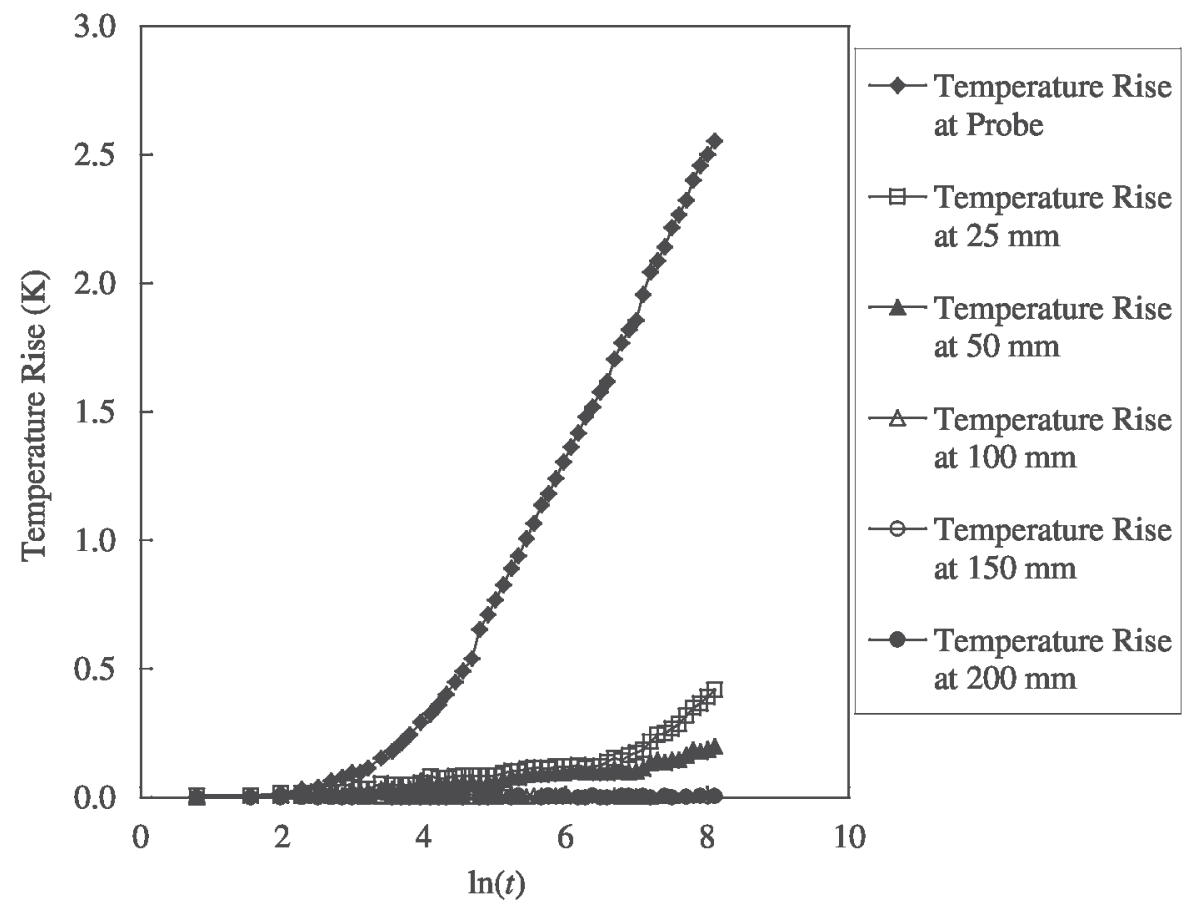

FIG. 5-Radius of influence testing (large probe in dry sand). 


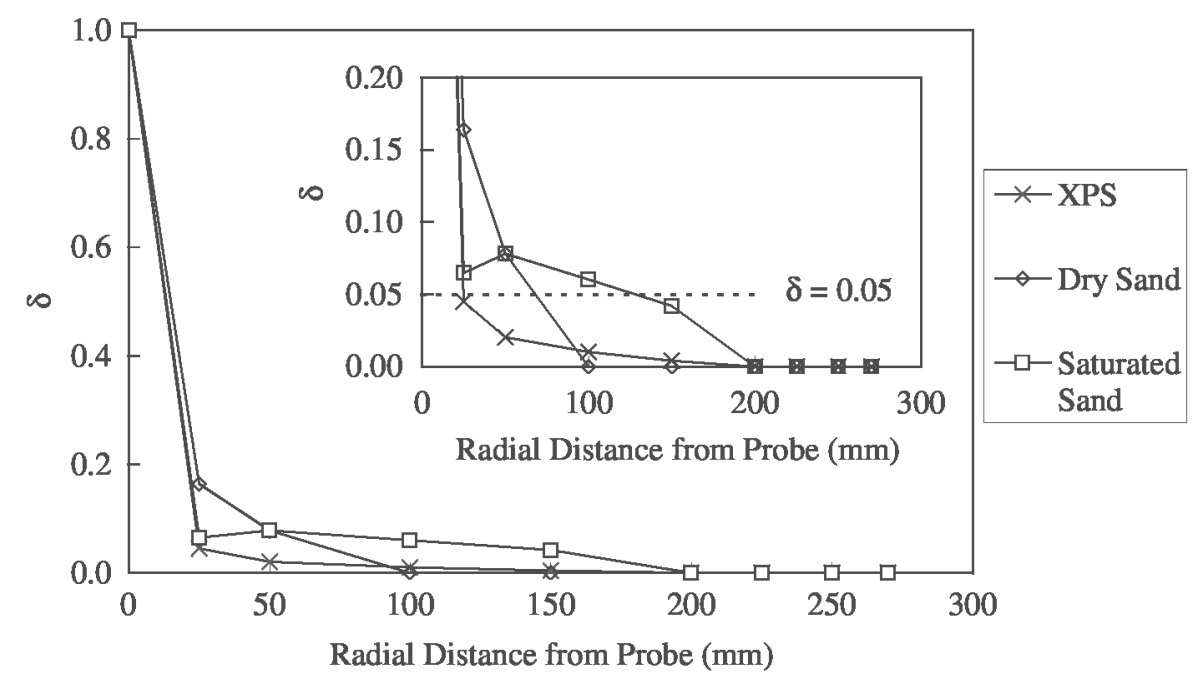

FIG. 6-Relative temperature increase in various materials.

TABLE 3-Relative temperature rise at various distances from the probe.

\begin{tabular}{|c|c|c|c|c|c|c|}
\hline \multirow{2}{*}{$\begin{array}{l}\text { Distance From Probe } \\
\text { mm }\end{array}$} & \multicolumn{2}{|c|}{ XPS } & \multicolumn{2}{|c|}{ Dry Sand } & \multicolumn{2}{|c|}{ Saturated Sand } \\
\hline & $T, \mathbf{K}$ & $\delta$ & $T, \mathbf{K}$ & $\delta$ & $T, \mathbf{K}$ & $\delta$ \\
\hline 0 & 7.950 & 1.00 & 2.55 & 1.00 & 1.79 & 1.00 \\
\hline 25 & 0.357 & 0.04 & 0.418 & 0.16 & 0.116 & 0.06 \\
\hline 50 & 0.162 & 0.02 & 0.199 & 0.08 & 0.14 & 0.08 \\
\hline 100 & 0.0806 & 0.01 & 0 & 0.00 & 0.108 & 0.06 \\
\hline 150 & 0.0317 & 0.00 & 0 & 0.00 & 0.075 & 0.04 \\
\hline 200 & $\ldots$ & $\ldots$ & 0 & 0.00 & $\ldots$ & .. \\
\hline
\end{tabular}

... Data not collected. 


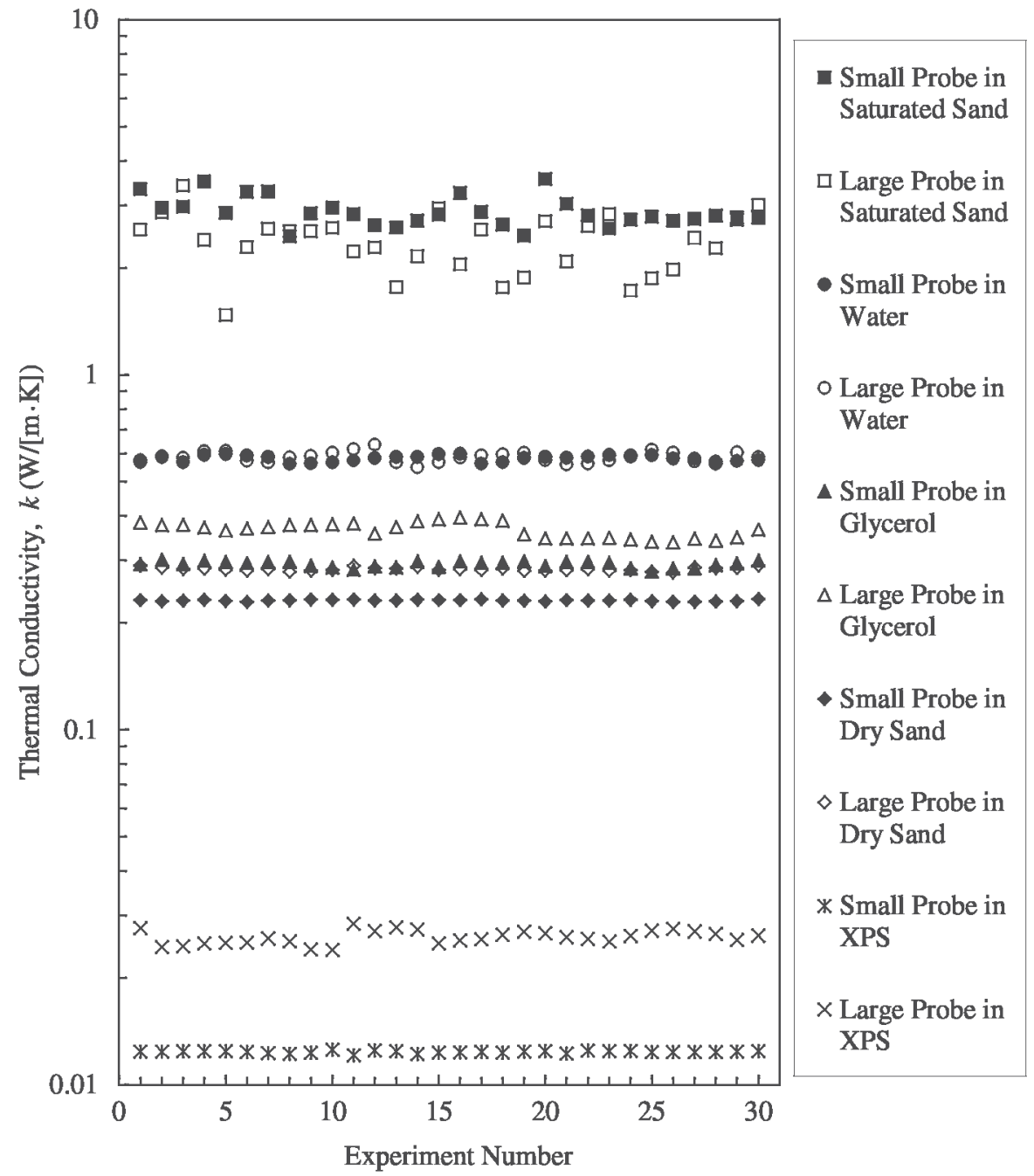

FIG. 7-Summary of calibration tests.

TABLE 4-Comparison of test results.

\begin{tabular}{|c|c|c|c|c|c|}
\hline \multirow[b]{2}{*}{ Material } & \multicolumn{2}{|c|}{$\begin{array}{c}\text { Baseline } \\
\text { Measurements }\end{array}$} & \multicolumn{2}{|c|}{$\begin{array}{l}\text { Large Probe } \\
\text { Measurments }\end{array}$} & \multirow[b]{2}{*}{$\begin{array}{c}k_{\text {small }} / \\
k_{\text {large }}\end{array}$} \\
\hline & $\begin{array}{c}k_{\text {small }}, \\
\mathrm{W} /[\mathrm{m} \cdot \mathrm{K}]\end{array}$ & $\operatorname{COV}^{1}$ & $\begin{array}{c}k_{\text {large }}, \\
\mathrm{W} /[\mathrm{m} \cdot \mathrm{K}]\end{array}$ & $\operatorname{COV}^{1}$ & \\
\hline XPS & 0.0124 & $0.7 \%$ & 0.0261 & $4.4 \%$ & 0.47 \\
\hline Dry Sand & 0.231 & $0.5 \%$ & 0.284 & $1.2 \%$ & 0.82 \\
\hline Glycerol & 0.293 & $2.1 \%$ & 0.366 & $5.1 \%$ & 0.80 \\
\hline Water & 0.581 & $2.1 \%$ & 0.586 & $3.6 \%$ & 0.99 \\
\hline Saturated Sand & 2.88 & $9.9 \%$ & 2.37 & $18.7 \%$ & 1.22 \\
\hline
\end{tabular}

${ }^{1}$ Coefficient of Variation. 


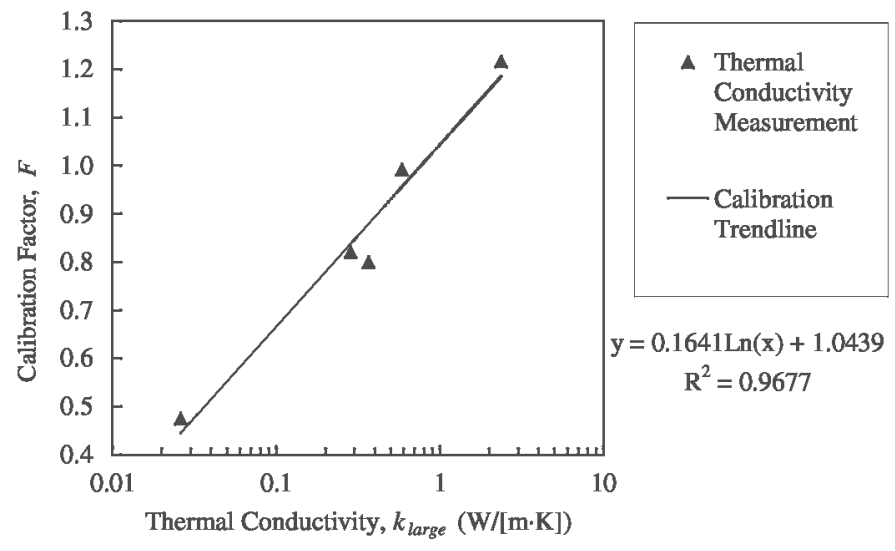

FIG. 8 Calibration curve.

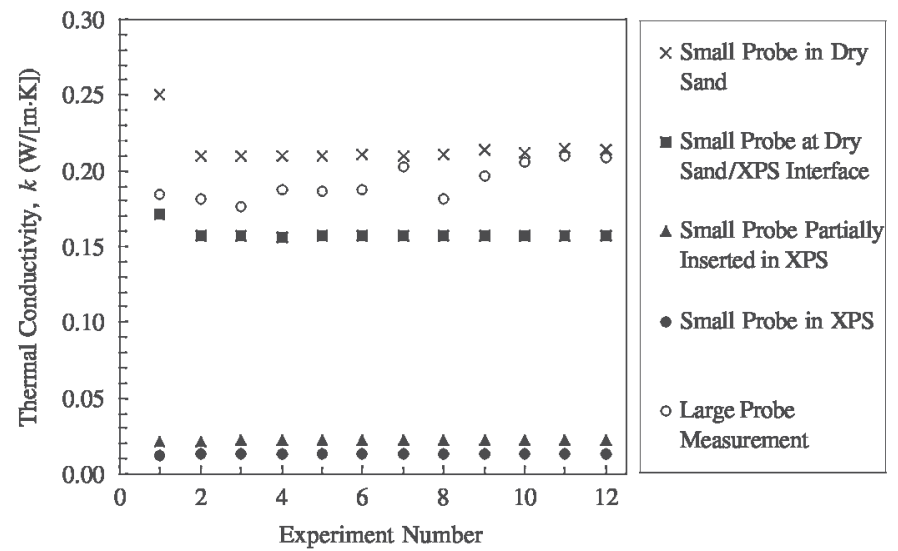

FIG. 9-Performance test 1 (dry Ottawa sand and XPS mixture).

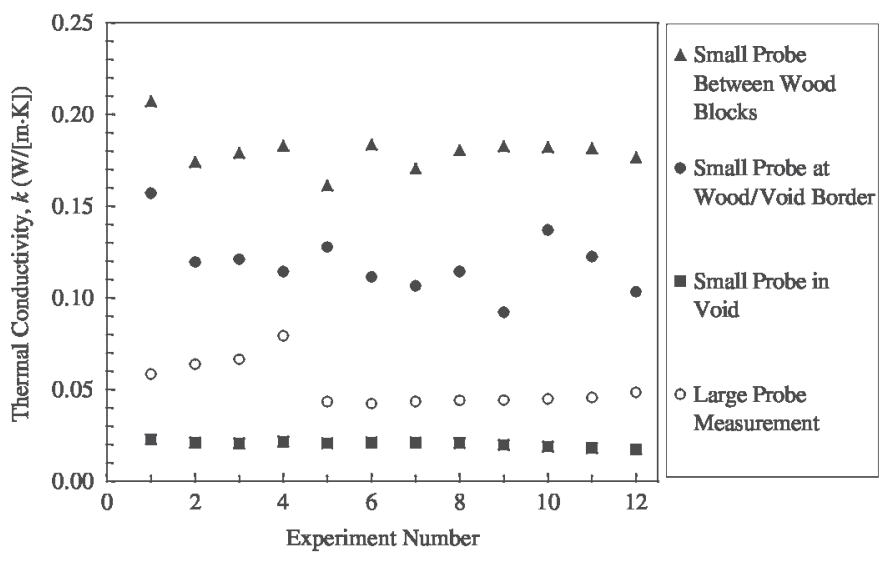

FIG. 11-Performance test 3 (wood blocks). 


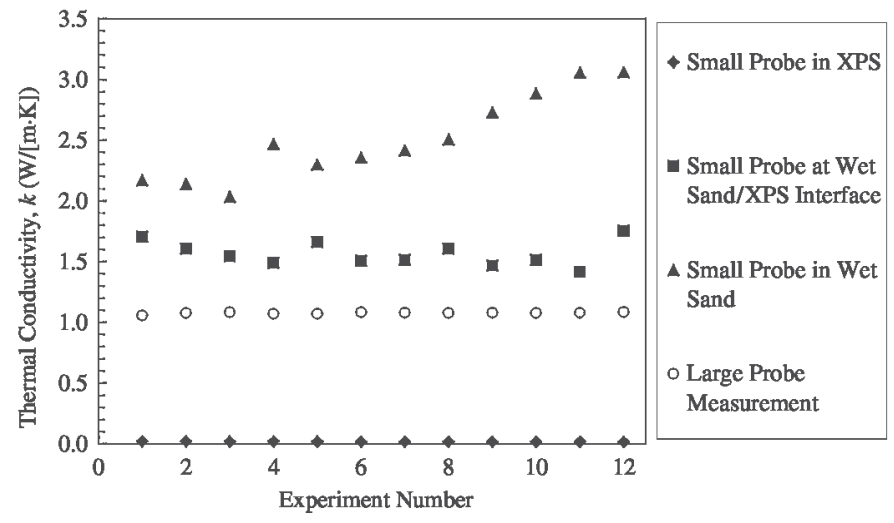

FIG. 10-Performance test 2 (saturated Ottawa sand and XPS mixture). 